\title{
Derivable Maps on Alternative Rings
}

\section{Aplicações Derivação sobre Anéis Alternativos}

\author{
Bruno L. M. Ferreira \\ Universidade Tecnológica Federal do Paraná - COECI-UTFPR, Guarapuava, PR \\ brunoferreira@utfpr.edu.br \\ Ruth Nascimento \\ Universidade Tecnológica Federal do Paraná - COECI-UTFPR, Guarapuava, PR \\ ruthnascimento@utfpr.edu.br
}

Abstract: $D$ is an application of an alternative ring in $\mathscr{R}$ satisfying $\mathscr{R} D(a b)=$ $D(a) b+a D(b)$ to the whole; $b \in \mathscr{R}$. With some conditions on $\mathscr{R}$, we show that $D$ additive.

Keywords: prime alternative ring; idempotent element; derivable map; additive map.

Resumo: Seja $D$ uma aplicação de um anel alternativo $\mathscr{R}$ em $\mathscr{R}$ satisfazendo $D(a b)=$ $D(a) b+a D(b)$ para todo $a, b \in \mathscr{R}$. Com algumas condições sobre $\mathscr{R}$, mostramos que $D$ é aditiva.

Palavras - Chave: anel alternativo primo; aplicação aditiva; aplicação derivação; elemento idempotente.

\section{Introduction}

In this paper, $\mathscr{R}$ will be a ring not necessarily associative or commutative. For $x, y, z \in \mathscr{R}$ we denote the associator by $(x, y, z)=(x y) z-x(y z)$.

A ring $\mathscr{R}$ is called $k$-torsion free if $k x=0$ implies $x=0$, for any $x \in \mathscr{R}$, where $k \in \mathbb{Z}, k>0$, prime if $I J \neq 0$ for any two nonzero ideals $I, J \subseteq \mathscr{R}$ and semiprime if it contains no nonzero ideal whose square is zero.

Recebido em 13/09/2013 - Aceito em 08/06/2014.

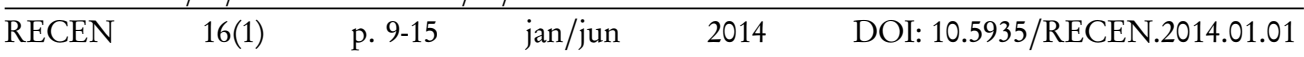


A ring $\mathscr{R}$ is said to be alternative if

$$
(x, x, y)=0=(y, x, x), \text { for all } x, y \in \mathscr{R},
$$

and flexible if

$$
(x, y, x)=0, \text { for all } x, y \in \mathscr{R} \text {. }
$$

One easily sees that any alternative ring is flexible.

Let $\mathscr{R}$ be an alternative ring (not necessarily with identity element) and let $a, b \in \mathscr{R}$. Recall that for a 3 -torsion free alternative ring $\mathscr{R}$ the following are equivalent:

(i) $\mathscr{R}$ is prime;

(ii) $(a \mathscr{R}) b \neq 0$ for any nonzero $a, b \in \mathscr{R}$;

(iii) $a(\mathscr{R} b) \neq 0$ for any nonzero $a, b \in \mathscr{R}$.

You can find the proof in [1]. A mapping $D: \mathscr{R} \rightarrow \mathscr{R}$ is derivable (multiplicative derivation) if $D(a b)=D(a) b+a D(b)$ for all $a, b \in \mathscr{R}$. Let us consider $\mathscr{R}$ an alternative ring and let us fix a nontrivial idempotent $e_{1} \in \mathscr{R}$, i.e, $e_{1}^{2}=e_{1} ; e_{1} \neq 0$ and $e_{1}$ is not an unity element. Let $e_{2}: \mathscr{R} \rightarrow \mathscr{R}$ and $e_{2}^{\prime}: \mathscr{R} \rightarrow \mathscr{R}$ be given by $e_{2} a=a-e_{1} a$ and $e_{2}^{\prime} a=a-a e_{1}$. We shall denote $e_{2}^{\prime} a$ by $a e_{2}$. Note that $\mathscr{R}$ need not have an identity element. The operation $x(1-y)$ for $x, y \in \mathscr{R}$ is understood as $x-x y$. It is easy to see that $\left(e_{i} a\right) e_{j}=e_{i}\left(a e_{j}\right)$ for all $a \in \mathscr{R}$ and $i, j=1,2$. Then $\mathscr{R}$ has a Peirce decomposition $\mathscr{R}=\mathscr{R}_{11} \oplus \mathscr{R}_{12} \oplus \mathscr{R}_{21} \oplus \mathscr{R}_{22}$, where $\mathscr{R}_{i j}=e_{i} \mathscr{R} e_{j}(i, j=1,2)$, satisfying the multiplicative relations:

(i) $\mathscr{R}_{i j} \mathscr{R}_{j l} \subseteq \mathscr{R}_{i l}(i, j, l=1,2)$;

(ii) $\mathscr{R}_{i j} \mathscr{R}_{i j} \subseteq \mathscr{R}_{j i}(i, j=1,2 ; i \neq j)$;

(iii) $\mathscr{R}_{i j} \mathscr{R}_{k l}=0$, if $j \neq k$ and $(i, j) \neq(k, l),(i, j, k, l=1,2)$;

(iv) $x_{i j}^{2}=0$ for all $x_{i j} \in \mathscr{R}_{i j}(i, j=1,2 ; i \neq j)$.

The study of the relationship between the multiplicative and the additive structures of a ring has become an interesting and active topic in ring theory. The present 
paper takes up the special case of an alternative ring. We investigate the problem of when a derivable map must be an additive map for the class of alternative rings.

\section{The main theorem}

We shall prove the following result:

Theorem 2.1: Let $\mathscr{R}$ be an alternative ring containing a non-trivial idempotent $e_{1}$ and $\mathscr{R}=\mathscr{R}_{11} \oplus \mathscr{R}_{12} \oplus \mathscr{R}_{21} \oplus \mathscr{R}_{22}$, the Peirce Decomposition of $\mathscr{R}$, relative to $e_{1}$, satisfying:

(i) If $\left(\left(e_{i} a\right) e_{j}\right) x_{j k}=0$ for all $x_{j k} \in \mathscr{R}_{j k}$, then $\left(\left(e_{i} a\right) e_{j}\right)=0$;

(ii) If $x_{i j}\left(\left(e_{j} a\right) e_{i}\right)=0$ for all $x_{i j} \in \mathscr{R}_{i j}$, then $\left(\left(e_{j} a\right) e_{i}\right)=0$,

for $i, j, k \in\{1,2\}$. If $D: \mathscr{R} \longrightarrow \mathscr{R}$ is a multiplicative derivation, then $D$ is additive.

The proof of the theorem is organized as a series of lemmas.

We begin with the following lemma with a simple proof.

Lemma 2.1: $D(0)=0$.

Proof. $D(0)=D(0.0)=D(0) 0+0 D(0)=0$.

Lemma 2.2: $D\left(a_{i i}+a_{j k}\right)=D\left(a_{i i}\right)+D\left(a_{j k}\right), j \neq k$.

Proof. If $i=1, j=1, k=2$, for any $t_{11} \in \mathscr{R}_{11}$, we compute $D\left(a_{11}+a_{12}\right) t_{11}+$ $a_{11} D\left(t_{11}\right)+D\left(a_{12} t_{11}\right)-D\left(a_{12}\right) t_{11}=D\left(a_{11}+a_{12}\right) t_{11}+\left(a_{11}+a_{12}\right) D\left(t_{11}\right)=D\left(\left(a_{11}+\right.\right.$ $\left.\left.a_{12}\right) t_{11}\right)=D\left(a_{11} t_{11}\right)=D\left(a_{11}\right) t_{11}+a_{11} D\left(t_{11}\right)$. By condition $(i)$ of Theorem 2.1, we have $\left[D\left(a_{11}+a_{12}\right)-\left(D\left(a_{11}\right)+D\left(a_{12}\right)\right)\right]_{11}=0$ and $\left[D\left(a_{11}+a_{12}\right)-\left(D\left(a_{11}\right)+D\left(a_{12}\right)\right)\right]_{21}=$ 0 . Now, for any $t_{22} \in \mathscr{R}_{22}$, we compute $D\left(a_{11}+a_{12}\right) t_{22}+a_{12} D\left(t_{22}\right)+D\left(a_{11} t_{22}\right)-$ $D\left(a_{11}\right) t_{22}=D\left(a_{11}+a_{12}\right) t_{22}+\left(a_{11}+a_{12}\right) D\left(t_{22}\right)=D\left(\left(a_{11}+a_{12}\right) t_{22}\right)=D\left(a_{12} t_{22}\right)=$ $D\left(a_{12}\right) t_{22}+a_{12} D\left(t_{22}\right)$. By condition $(i)$ of Theorem 2.1, we have $\left[D\left(a_{11}+a_{12}\right)-\right.$ $\left.\left(D\left(a_{11}\right)+D\left(a_{12}\right)\right)\right]_{12}=0$ and $\left[D\left(a_{11}+a_{12}\right)-\left(D\left(a_{11}\right)+D\left(a_{12}\right)\right)\right]_{22}=0$. Thus, $D\left(a_{11}+\right.$ $\left.a_{12}\right)=D\left(a_{11}\right)+D\left(a_{12}\right)$.

The proof of the remaining cases is similar. 
Lemma 2.3: $D\left(a_{12}+a_{21}\right)=D\left(a_{12}\right)+D\left(a_{21}\right)$.

Proof. For any $t_{11} \in \mathscr{R}_{11}$, we compute $D\left(a_{12}+a_{21}\right) t_{11}-D\left(a_{12}\right) t_{11}+D\left(a_{21} t_{11}\right)-$ $D\left(a_{21}\right) t_{11}=D\left(a_{12}+a_{21}\right) t_{11}+\left(a_{12}+a_{21}\right) D\left(t_{11}\right)=D\left(\left(a_{12}+a_{21}\right) t_{11}\right)=D\left(a_{21} t_{11}\right)$. By condition $(i)$ of Theorem 2.1, we have $\left[D\left(a_{12}+a_{21}\right)-\left(D\left(a_{12}\right)+D\left(a_{21}\right)\right)\right]_{11}=0$ and $\left[D\left(a_{12}+a_{21}\right)-\left(D\left(a_{12}\right)+D\left(a_{21}\right)\right)\right]_{21}=0$. Similarly, replacing $t_{11}$ by $t_{22}$ we obtain $\left[D\left(a_{12}+a_{21}\right)-\left(D\left(a_{12}\right)+D\left(a_{21}\right)\right)\right]_{12}=0$ and $\left[D\left(a_{12}+a_{21}\right)-\left(D\left(a_{12}\right)+D\left(a_{21}\right)\right)\right]_{22}=0$. $\diamond$

Lemma 2.4: $\quad$ (i) $D\left(a_{12}+b_{12} c_{22}\right)=D\left(a_{12}\right)+D\left(b_{12} c_{22}\right)$,

(ii) $D\left(a_{21}+b_{22} c_{21}\right)=D\left(a_{21}\right)+D\left(b_{22} c_{21}\right)$.

Proof. (i) We have $\left(e_{1}+b_{12}\right)\left(a_{12}+c_{22}\right)=a_{12}+b_{12} a_{12}+b_{12} c_{22}$. Then by Lemma 2.2 we have $D\left[\left(e_{1}+b_{12}\right)\left(a_{12}+c_{22}\right)\right]=D\left(e_{1}+b_{12}\right)\left(a_{12}+c_{22}\right)+\left(e_{1}+b_{12}\right) D\left(a_{12}+c_{22}\right)=$ $\left(D\left(e_{1}\right)+D\left(b_{12}\right)\right)\left(a_{12}+c_{22}\right)+\left(e_{1}+b_{12}\right)\left(D\left(a_{12}\right)+D\left(c_{22}\right)\right)=D\left(e_{1} a_{12}\right)+D\left(e_{1} c_{22}\right)+$ $D\left(b_{12} a_{12}\right)+D\left(b_{12} c_{22}\right)$. By other hand, by Lemma $2.3, D\left(a_{12}+b_{12} a_{12}+b_{12} c_{22}\right)=$ $D\left(b_{12} a_{12}\right)+D\left(a_{12}+b_{12} c_{22}\right)$. Thus, $D\left(a_{12}+b_{12} c_{22}\right)=D\left(a_{12}\right)+D\left(b_{12} c_{22}\right)$.

(ii) It is analogous, by using the relation $\left(a_{21}+b_{22}\right)\left(e_{1}+c_{21}\right)=a_{21}+a_{21} c_{21}+b_{22} c_{21}$. $\diamond$

Lemma 2.5: $\quad$ (i) $D\left(a_{12}+b_{12}\right)=D\left(a_{12}\right)+D\left(b_{12}\right)$,

(ii) $D\left(a_{21}+b_{21}\right)=D\left(a_{21}\right)+D\left(b_{21}\right)$.

Proof. (i) For any $t_{22} \in \mathscr{R}_{22}$, by Lemma 2.4 part $(i)$, we have $D\left(a_{12}\right) t_{22}+a_{12} D\left(t_{22}\right)+$ $D\left(b_{12}\right) t_{22}+b_{12} D\left(t_{22}\right)=D\left(a_{12} t_{22}\right)+D\left(b_{12} t_{22}\right)=D\left(a_{12} t_{22}+b_{12} t_{22}\right)=D\left(\left(a_{12}+\right.\right.$ $\left.\left.b_{12}\right) t_{22}\right)=D\left(a_{12}+b_{12}\right) t_{22}+\left(a_{12}+b_{12}\right) D\left(t_{22}\right)$. Thus, by $(i)$ of the Theorem 2.1, $\left[D\left(a_{12}+b_{12}\right)-\left(D\left(a_{12}\right)+D\left(b_{12}\right)\right)\right]_{12}=0$ and $\left[D\left(a_{12}+b_{12}\right)-\left(D\left(a_{12}\right)+D\left(b_{12}\right)\right)\right]_{22}=0$. Now, for any $t_{11} \in \mathscr{R}_{11}$, we have $D\left(a_{12}+b_{12}\right) t_{11}+\left(a_{12}+b_{12}\right) D\left(t_{11}\right)=D\left(\left(a_{12}+\right.\right.$ $\left.\left.b_{12}\right) t_{11}\right)=D(0)=0=D(0)+D(0)=D\left(a_{12} t_{11}\right)+D\left(b_{12} t_{11}\right)=D\left(a_{12}\right) t_{11}+a_{12} D\left(t_{11}\right)+$ $D\left(b_{12}\right) t_{11}+b_{12} D\left(t_{11}\right)$. So, by $(i)$ of Theorem 2.1, $\left[D\left(a_{12}+b_{12}\right)-\left(D\left(a_{12}\right)+D\left(b_{12}\right)\right)\right]_{11}=$ 0 and $\left[D\left(a_{12}+b_{12}\right)-\left(D\left(a_{12}\right)+D\left(b_{12}\right)\right)\right]_{21}=0$.

(ii) For any $t_{22} \in \mathscr{R}_{22}$ by Lemma 2.4 part (ii), we have $D\left(t_{22}\left(a_{21}+b_{21}\right)\right)=$ $D\left(t_{22} a_{21}+t_{22} b_{21}\right)=D\left(t_{22} a_{21}\right)+D\left(t_{22} b_{21}\right)=D\left(t_{22}\right) a_{21}+t_{22} D\left(a_{21}\right)+D\left(t_{22}\right) b_{21}+$ 
$t_{22} D\left(b_{21}\right)=t_{22}\left(D\left(a_{21}\right)+D\left(b_{21}\right)\right)+D\left(t_{22}\right)\left(a_{21}+b_{21}\right)$. By other hand, $D\left(t_{22}\left(a_{21}+\right.\right.$ $\left.\left.b_{21}\right)\right)=D\left(t_{22}\right)\left(a_{21}+b_{21}\right)+t_{22} D\left(a_{21}+b_{21}\right)$. Thus, by condition (ii) of Theorem 2.1, $\left[D\left(a_{21}+b_{21}\right)-\left(D\left(a_{21}\right)+D\left(b_{21}\right)\right)\right]_{21}=0$ and $\left[D\left(a_{21}+b_{21}\right)-\left(D\left(a_{21}\right)+D\left(b_{21}\right)\right)\right]_{22}=0$. Now, for any $t_{11} \in \mathscr{R}_{11}, D\left(t_{11}\right) a_{21}+t_{11} D\left(a_{21}\right)+D\left(t_{11}\right) b_{21}+t_{11} D\left(b_{21}\right)=D\left(t_{11} a_{21}\right)+$ $D\left(t_{11} b_{21}\right)=0=D(0)=D\left(t_{11}\left(a_{21}+b_{21}\right)\right)=D\left(t_{11}\right)\left(a_{21}+b_{21}\right)+t_{11} D\left(a_{21}+b_{21}\right)$. By condition (ii) of Theorem 2.1, $\left[D\left(a_{21}+b_{21}\right)-\left(D\left(a_{21}\right)+D\left(b_{21}\right)\right)\right]_{11}=0$ and $\left[D\left(a_{21}+b_{21}\right)-\left(D\left(a_{21}\right)+D\left(b_{21}\right)\right)\right]_{12}=0$.

Lemma 2.6: (i) $D\left(a_{11}+b_{11}\right)=D\left(a_{11}\right)+D\left(b_{11}\right)$,

(ii) $D\left(a_{22}+b_{22}\right)=D\left(a_{22}\right)+D\left(b_{22}\right)$.

Proof. (i) For any $t_{22} \in \mathscr{R}_{22}$, we have $D\left(a_{11}+b_{11}\right) t_{22}+\left(a_{11}+b_{11}\right) D\left(t_{22}\right)=$ $D\left(\left(a_{11}+b_{11}\right) t_{22}\right)=0=D(0)=D(0)+D(0)=D\left(a_{11} t_{22}\right)+D\left(b_{11} t_{22}\right)=D\left(a_{11}\right) t_{22}+$ $a_{11} D\left(t_{22}\right)+D\left(b_{1} 1\right) t_{22}+b_{11} D\left(t_{22}\right)$. So, by condition $(i)$ of Theorem 2.1, $\left[D\left(a_{11}+\right.\right.$ $\left.\left.b_{11}\right)-\left(D\left(a_{11}\right)+D\left(b_{11}\right)\right)\right]_{12}=0$ and $\left[D\left(a_{11}+b_{11}\right)-\left(D\left(a_{11}\right)+D\left(b_{11}\right)\right)\right]_{22}=0$. Now, for any $t_{12} \in \mathscr{R}_{12}$ by Lemma 2.5 part $(i)$, we have $D\left(a_{11}+b_{11}\right) t_{12}+\left(a_{11}+b_{11}\right) D\left(t_{12}\right)=$ $D\left(\left(a_{11}+b_{11}\right) t_{12}\right)=D\left(a_{11} t_{12}\right)+D\left(b_{11} t_{12}\right)=D\left(a_{11}\right) t_{12}+a_{11} D\left(t_{12}\right)+D\left(b_{11}\right) t_{12}+$ $b_{11} D\left(t_{12}\right)$. Thus, by $(i)$ of the Theorem, we have $\left[D\left(a_{11}+b_{11}\right)-\left(D\left(a_{11}\right)+D\left(b_{11}\right)\right)\right]_{11}=$ 0 and $\left[D\left(a_{11}+b_{11}\right)-\left(D\left(a_{11}\right)+D\left(b_{11}\right)\right)\right]_{21}=0$.

(ii) It is analogous.

Lemma 2.7: $D\left(a_{11}+b_{12}+c_{21}+d_{22}\right)=D\left(a_{11}\right)+D\left(b_{12}\right)+D\left(c_{21}\right)+D\left(d_{22}\right)$.

Proof. For any $t_{11} \in \mathscr{R}_{11}$ by Lemma 2.2, we have $D\left(a_{11}+b_{12}+c_{21}+d_{22}\right) t_{11}+$ $\left(a_{11}+b_{12}+c_{21}+d_{22}\right) D\left(t_{11}\right)=D\left(\left(a_{11}+b_{12}+c_{21}+d_{22}\right) t_{11}\right)=D\left(a_{11} t_{11}+c_{21} t_{11}\right)=$ $D\left(a_{11} t_{11}\right)+D\left(c_{21} t_{11}\right)=D\left(a_{11}\right) t_{11}+a_{11} D\left(t_{11}\right)+D\left(c_{21}\right) t_{11}+c_{21} D\left(t_{11}\right)+0+0=$ $D\left(a_{11}\right) t_{11}+a_{11} D\left(t_{11}\right)+D\left(c_{21}\right) t_{11}+c_{21} D\left(t_{11}\right)+D\left(b_{12} t_{11}\right)+D\left(d_{22} t_{11}\right)=D\left(a_{11}\right) t_{11}+$ $a_{11} D\left(t_{11}\right)+D\left(c_{21}\right) t_{11}+c_{21} D\left(t_{11}\right)+D\left(b_{12}\right) t_{11}+b_{12} D\left(t_{11}\right)+D\left(d_{22}\right) t_{11}+d_{22} D\left(t_{11}\right)$. Thus, by condition $(i)$ of the Theorem 2.1 , we have, $\left[D\left(a_{11}+b_{12}+c_{21}+d_{22}\right)-\right.$ $\left.\left(D\left(a_{11}\right)+D\left(b_{12}\right)+D\left(c_{21}\right)+D\left(d_{22}\right)\right)\right]_{11}=0$ and $\left[D\left(a_{11}+b_{12}+c_{21}+d_{22}\right)-\left(D\left(a_{11}\right)+\right.\right.$ $\left.\left.D\left(b_{12}\right)+D\left(c_{21}\right)+D\left(d_{22}\right)\right)\right]_{21}=0$. Now, for any $t_{22} \in \mathscr{R}_{22}$ by Lemma 2.2, we have $D\left(a_{11}+b_{12}+c_{21}+d_{22}\right) t_{22}+\left(a_{11}+b_{12}+c_{21}+d_{22}\right) D\left(t_{22}\right)=D\left(\left(a_{11}+b_{12}+c_{21}+\right.\right.$ $\left.\left.d_{22}\right) t_{22}\right)=D\left(b_{12} t_{22}+d_{22} t_{22}\right)=D\left(b_{12} t_{22}\right)+D\left(d_{22} t_{22}\right)=D\left(b_{12}\right) t_{22}+b_{12} D\left(t_{22}\right)+$ 


$$
\begin{aligned}
& D\left(d_{22}\right) t_{22}+d_{22} D\left(t_{22}\right)+0+0=D\left(b_{12}\right) t_{22}+b_{12} D\left(t_{22}\right)+D\left(d_{22}\right) t_{22}+d_{22} D\left(t_{22}\right)+ \\
& D\left(a_{11} t_{22}\right)+D\left(c_{21} t_{22}\right)=D\left(b_{12}\right) t_{22}+b_{12} D\left(t_{22}\right)+D\left(d_{22}\right) t_{22}+d_{22} D\left(t_{22}\right)+D\left(a_{11}\right) t_{22}+ \\
& a_{11} D\left(t_{22}\right)+D\left(c_{21}\right) t_{22}+c_{21} D\left(t_{22}\right) . \text { So, by condition }(i) \text { of Theorem 2.1, we have, } \\
& {\left[D\left(a_{11}+b_{12}+c_{21}+d_{22}\right)-\left(D\left(a_{11}\right)+D\left(b_{12}\right)+D\left(c_{21}\right)+D\left(d_{22}\right)\right)\right]_{12}=0 \text { and }\left[D \left(a_{11}+\right.\right.} \\
& \left.\left.b_{12}+c_{21}+d_{22}\right)-\left(D\left(a_{11}\right)+D\left(b_{12}\right)+D\left(c_{21}\right)+D\left(d_{22}\right)\right)\right]_{22}=0 .
\end{aligned}
$$

Now we are ready to prove our main result.

Proof of the Theorem 2.1: For any $a, b \in \mathscr{R}$, we write $a=a_{11}+a_{12}+a_{21}+a_{22}$ and $b=b_{11}+b_{12}+b_{21}+b_{22}$. Applying Lemmas 2.5, 2.6 and 2.7, we have

$$
\begin{aligned}
D(a+b)= & D\left(\left(a_{11}+a_{12}+a_{21}+a_{22}\right)+\left(b_{11}+b_{12}+b_{21}+b_{22}\right)\right) \\
= & D\left(a_{11}+b_{11}+a_{12}+b_{12}+a_{21}+b_{21}+a_{22}+b_{22}\right) \\
= & D\left(a_{11}+b_{11}\right)+D\left(a_{12}+b_{12}\right)+D\left(a_{21}+b_{21}\right)+D\left(a_{22}+b_{22}\right) \\
= & D\left(a_{11}\right)+D\left(b_{11}\right)+D\left(a_{12}\right)+D\left(b_{12}\right)+D\left(a_{21}\right)+D\left(b_{21}\right)+D\left(a_{22}\right)+ \\
& D\left(b_{22}\right) \\
= & D\left(a_{11}+a_{12}+a_{21}+a_{22}\right)+D\left(b_{11}+b_{12}+b_{21}+b_{22}\right)=D(a)+D(b) .
\end{aligned}
$$

Therefore, $D$ is additive.

Corollary 2.1: Let $\mathscr{R}$ be a 3-torsion free prime alternative ring containing a nontrivial idempotent and a multiplicative derivation $D: \mathscr{R} \longrightarrow \mathscr{R}$, then $D$ is additive.

Definition 2.1: Let $\mathscr{R}$ be an alternative ring and $d$ be a map from $\mathscr{R}$ into itself. We call $d$ a Jordan derivable map if $d(a b+b a)=d(a) b+b d(a)+d(b) a+b d(a)$ for all $a, b \in \mathscr{R}$.

Corollary 2.2: Let $\mathscr{R}$ be an alternative ring containing a nontrivial idempotent, satisfying (i), (ii) of the Theorem 2.1 and a multiplicative derivation, $D: \mathscr{R} \longrightarrow \mathscr{R}$, then $D$ is a additive Jordan derivable map. 


\section{Referências}

[1] I.R. Hentzel, E. Kleinfeld; H.F. Smith, Alternative Rings with Idempotent, J Algebra, v. 64, p. 325-335, 1980.

[2] DAIF, M. When is a multiplicative derivation additive ?, Int J Math Math Sci, v. 14, p. 615-618, 1991.

[3] J. C. Motta Ferreira; H. Guzzo Jr. Jordan elementary maps on alternative rings. Comunications in Algebra, v.42, p. 779-794, 2014. 